SLA C-PUB-1395

(T)

\title{
ASYMPTOTIC BEHAVIOR OF FORM FACTORS FOR
}

TWO- AND THREE-BODY BOUND STATES

C. Alabiso and G Schierholz

\section{Errata}

Page 1, line 6 (of the text)

Page 1, line 9 (of the text)

Page 3 , line 22

Page 10 , formula (20)

Page 1 , line 10 (of the text)

$$
\left.\overrightarrow{(k}^{2}\right)^{-\theta}(0<\theta \leq 1)
$$

Corrigenda

$(|\overrightarrow{\mathrm{k}}|)^{-1-\theta}(0<\theta)$

$\left.\mathrm{F}_{2} \overrightarrow{\mathrm{q}}^{2}\right) \approx(|\overrightarrow{\mathrm{q}}|)^{-2-2 \theta}\left(0<\theta \leq \frac{1}{2}\right)$,

$\left.\mathrm{F}_{2} \overrightarrow{\mathrm{q}}^{2}\right) \simeq(\overrightarrow{\mid q} \mid)^{-2.5-\theta}\left(\frac{1}{2} \leq \theta\right)$,

$$
\left.\left.\mathrm{F}_{3} \overrightarrow{\mathrm{q}}^{2}\right) \simeq(|\overrightarrow{\mathrm{q}}|)^{-5-\theta}, \quad \mathrm{F}_{3} \dot{\overrightarrow{\mathrm{q}}}^{2}\right) \simeq(|\overrightarrow{\mathrm{q}}|)^{-5-2 \theta},
$$




\title{
ASYMPTOTIC BEHAVIOR OF FORM FACTORS FOR
}

\author{
TWO- AND THREE-BODY BOUND STATES* \\ C. Alabiso† and G. Schierholz $\dagger \dagger$ \\ Stanford Linear Accelerator Center \\ Stanford University, Stanford, Calif. 94305
}

\begin{abstract}
The asymptotic power behavior of the electromagnetic form factors are examined for two- and three-body s-wave bound states both relativisitc and nonrelativistic. In the nonrelativistic case we consider local and separable two-body potentials and we make use of the Faddeev equations in order to define the three-body bound states. For local potentials which behave as $\left(\vec{k}^{2}\right)^{-\theta}(0<\theta \leq 1)$ for large momentum transfer, we obtain for the asymptotic power behavior of the form factors of the two- and three-body bound states $\mathrm{F}_{2}\left(\vec{q}^{2}\right) \simeq(|\vec{q}|)^{-3-\theta}$ and $\left.\mathrm{F}_{3} \overrightarrow{(\vec{q}}^{2}\right) \simeq(|\vec{q}|)^{-6-2 \theta}$, respectively. For separable potentials $V=g(|\vec{k}|) g\left(\left|\overrightarrow{k^{1}}\right|\right)$ and $g(|\vec{k}|) \simeq(|\vec{k}|)^{-\frac{1}{2}-\theta}$ we find $\left.F_{2} \vec{q}^{2}\right) \simeq(|\vec{q}|)^{-2-\theta}$ and $\left.\mathrm{F}_{3} \overrightarrow{\mathrm{q}}^{2}\right) \simeq(|\overrightarrow{\mathrm{q}}|)^{-5-\theta}$, respectively. For the relativistic case, we consider the two- and three-body Bethe-Salpeter equation in the ladder approximation. We treat the spin zero case only but we believe that our final conclusions will not be affected by the introduction of spin $1 / 2$ particle. With an interaction which behaves as $\left(\mathrm{k}^{2}\right)^{-\theta}$ at large momentum transfer, we obtain $F_{2}\left(q^{2}\right) \simeq\left(q^{2}\right)^{-1-\theta}$ and $\mathrm{F}_{3}\left(\mathrm{q}^{2}\right) \simeq\left(\mathrm{q}^{2}\right)^{-2-2 \theta}$
\end{abstract}

(Submitted to Phys. Rev.)

* Work supported in part by the U.S. Atomic Energy Commission.

$\dagger$ On leave of absence of Istituto di Fisica dell' Università di Parma, Parma, Italy. Consiglio Nazionale della Ricerche fellow.

t† On leave of absence and present address: II. Institut für Theoretische Physik der Universität, Hamburg, Germany. 


\section{INTRODUCTION, RESULTS AND CONCLUSIONS}

The evaluation of the electromagnetic hadron form factors has been a constant task for the last five years. ${ }^{1-6}$ It soon became clear that the large momentum transfer behavior of the form factors provides a powerful mean of studying the constituents of the hadrons and their dynamics. It is by now well accepted that the behaviors $F_{\pi}\left(q^{2}\right) \simeq \frac{1}{q^{2}}$ and $F_{1 p}\left(q^{2}\right) \simeq \frac{1}{\left(q^{2}\right)^{2}}$ are compatible with the experiments. ${ }^{7}$ This fact suggests that the pion and the nucleon certainly are of a different nature as far as the electromagnetic interaction are concerned. It seems also to suggest that the pion is less composite than the nucleon because of the faster decrease of the proton form factor. Recently, the previous behaviors have been derived from the minimal quark structure of the pion and the proton; 8,9 so far, however, the three-particle bound state has not been treated in a convincing way and this leaves the question open whether the underlying twoand three-particle structure can explain the different behavior of the two form factors.

It is the aim of this paper to investigate the large $q^{2}$ behavior of the form factors of the two- and three-particle s-wave bound states in a systematic way, both in relativistic and nonrelativistic theories. Throughout the paper we consider power behaviors only, neglecting possible logarithmic factors. Here, in a first approach, we restrict ourselves to spinless constituents. We do not believe that the case of spin $1 / 2$ constituents makes a real difference on our final conclusions. This case will be discussed elsewhere. ${ }^{10}$

We shall consider the potential scattering case (II) for two main reasons. First, because many features of composite particle models can be explained by means of the nonrelativistic quark model ${ }^{11}$ moreover, the Bethe-Salpeter equation in the ladder approximation reduces to a nonrelativistic form in the 
large momenta limit, as it can be recovered from various (equivalent) threedimensional equations. ${ }^{12-15}$ The second good reason for studying the potential theory is the firm mathematical ground on which the nonrelativistic threeparticle theory in the form of the Faddeev equations ${ }^{16}$ is based (we do not consider three-particle forces).

For both two- and three-particle cases, we shall assume the two-body local potentials $V(|\overrightarrow{\mathrm{k}}|) \underset{|\overrightarrow{\mathrm{k}}|}{\simeq}(|\overrightarrow{\mathrm{k}}|)^{-1-\theta}, \theta>0$, and the separable potentials $\mathrm{V}\left(\overrightarrow{\mathrm{k}}, \overrightarrow{\mathrm{k}^{\prime}}\right)=\mathrm{g}(|\overrightarrow{\mathrm{k}}|) \mathrm{g}\left(\left|\overrightarrow{\mathrm{k}^{\prime}}\right|\right)$ with $\mathrm{g}(|\overrightarrow{\mathrm{k}}|) \underset{|\overrightarrow{\mathrm{k}}| \rightarrow \infty}{\simeq}(|\overrightarrow{\mathrm{k}}|)^{-1 / 2-\theta}, \theta>0$. Our choice of the potentials is determined by simple reasons. For the local potentials, the limiting behavior $(|\overrightarrow{\mathrm{k}}|)^{-1}$ is characteristic of the singular potential $\left(-\lambda / \mathrm{r}^{2}\right)$ which produces the unpleasant feature of a wave function fall-off depending on the coupling constant. 17,18 On the other hand, an even more singular potential gives rise to the exponential decrease of the wave function and of the form factor both, ${ }^{19}$ and this does not seem to be the physical case. As far as the separable potential is concerned, the choice $\theta>0$ is imposed by the very existence of scattering processes. The use of nonlocal potentials is suggested both by the existence of tensor forces in the spin $1 / 2$ case, and by the structure of the relativistic potential as recovered in the three-dimensional version of the BetheSalpeter equation. $10,12-15$

Our results are as follows. For the two-body and three-body bound states form factors we obtain $\mathrm{F}_{2}\left(\overrightarrow{\mathrm{q}}^{2}\right) \simeq(|\overrightarrow{\mathrm{q}}|)^{-3-\theta}$ and $\mathrm{F}_{3}\left(\overrightarrow{\mathrm{q}}^{2}\right) \simeq(\mid \overrightarrow{\mathrm{q} \mid})^{-6-2 \theta}$, with local potentials, whereas we obtain $\mathrm{F}_{2}\left(\overrightarrow{\mathrm{q}}^{2}\right) \simeq(\mid \overrightarrow{\mathrm{q} \mid})^{-2-\theta}$ and $\mathrm{F}_{3}\left(\overrightarrow{\mathrm{q}}^{2}\right) \simeq(\mid \overrightarrow{\mathrm{q} \mid})^{-5-2 \theta}$ with separable potentials。

In the framework of relativistic theories, we consider (III) the s-wave bound states of two and three particles described by the two-body Bethe-Salpeter equation in the ladder approximation (III A) and by the relativistic Faddeev 
equations (III B) ${ }^{12-15,20}$ We shall assume a two-body interaction of the form $\mathrm{V}(\mathrm{k})_{\mathrm{k} \longrightarrow \infty}^{2} \simeq\left(\mathrm{k}^{2}\right)^{-\theta}, \theta>0$. Our interactions correspond to the $\lambda \varphi^{3}$ theory for $\theta=1$ and to the $\lambda \varphi^{4}$ theory for the limiting case $\theta=0$. For the latter case it has been proved ${ }^{2,4,18}$ that the high momentum transfer behavior of the twobody wave functions and form factors depends on the coupling constant, as in the singular $\left(-\lambda / r^{2}\right)$ potential.

Our results for the asymptotic behavior of the two- and three-body form factors are $\mathrm{F}_{2}\left(\mathrm{q}^{2}\right) \simeq\left(\mathrm{q}^{2}\right)^{-1-\theta}, \mathrm{F}_{3}\left(\mathrm{q}^{2}\right) \simeq\left(\mathrm{q}^{2}\right)^{-2-2 \theta^{\circ}}$.

Since the $\lambda \varphi^{4}$ theory leads to that strange dependence on the coupling constant, we define the physical form factors as given by our superrenormalizable interaction in the limit $\theta \rightarrow 0$; the asymptotic behavior of our "pion" and "nucleon" form factors turns out to be $\left(q^{2}\right)^{-1}$ and $\left(q^{2}\right)^{-2}$, respectively.

The spin $\frac{1}{2}$ constituents, which are more interesting for the physical situation, present some technical difficulties: apart from the complicate spinstructure of the three-body wave function, there appears a delicate region of integration so that one has to be more careful than in the spin zero case. However, we do not agree with Ref. 4, note 25, where the author claims that the consistency argument, widely applied in our paper, does not work for superrenormalizable interactions.

Finally, it is worthwhile to remark that our results are in agreement with the predictions given in Ref。 8 and in Ref。9. Furthermore, our wave functions turn out to be integrable as it was assumed in Ref. 8 as a crucial hypothesis. 


\section{POTENTIAL SCATTERING}

In the framework of a potential scattering theory we shall discuss the asymptotic behavior of the two- and three-body bound states form factors at large momentum transfer; we shall consider s-wave bound states only. Furthermore, in order to simplify things, we shall always assume that only one particle is charged. Let us start with the two-body case. Here the charge form factor reads:

$$
\left.F_{2} \vec{q}^{2}\right)=\int d \overrightarrow{q^{\prime}} \psi^{*}\left(\overrightarrow{q^{\prime}}\right) \psi\left(\overrightarrow{q^{\prime}}-\vec{q}\right)
$$

where the wave function $\psi$ satisfies the homogeneous Schroedinger equation:

$$
\psi(\vec{q})=\frac{1}{q^{2}-E} \int d \vec{k} V(\vec{q}-\vec{k}) \psi(\vec{k})
$$

If we now consider a central potential which behaves at large $|\vec{k}|$ as

$$
\mathrm{V}(\overrightarrow{\mathrm{k}}) \simeq \frac{1}{|\overrightarrow{\mathrm{k}}|} \mid+\theta, \quad \theta>0,
$$

we get the following behavior for $\psi$ and $\mathrm{F}_{2}$ :

$$
\psi\left(\overrightarrow{\mathrm{q})} \underset{|\overrightarrow{\mathrm{q}}|}{\simeq} \frac{1}{|\overrightarrow{\mathrm{q}}|^{3+\theta}}, \quad \mathrm{F}_{2} \overrightarrow{(\vec{q}}^{2}\right) \quad \underset{|\overrightarrow{\mathrm{q}}|}{\simeq} \underset{\rightarrow \infty}{\simeq|\overrightarrow{\mathrm{q}}|^{3+\theta}}
$$

In the limiting case $\theta=0$ which corresponds to the potential $\left(-\lambda / r^{2}\right)$ the form factor behaves like $(|\vec{q}|)^{-2-2 \sqrt{\frac{1}{4}-\lambda}}(0<\lambda<1 / 4)$ with an unpleasant dependence on the coupling constant $\lambda$ (a similar phenomenon occurs in the Bethe-Salpeter equation $^{2,4,18}$ ). With an even more singular potential, the wave function and hence the form factor) becomes exponentially decreasing. ${ }^{19}$ In conclusion, with a central potential, the desired $1 / q^{2}$ behavior of the "pion" form factor is achieved only with the singular potential $\left(-\lambda / r^{2}\right)$ and only in the particular limit $\lambda \rightarrow 1 / 4$. 
For the three-body case, we consider the Faddeev equations ${ }^{16}$ with minor changes in the notations. Let $\overrightarrow{p_{1}}, \overrightarrow{p_{2}}, \overrightarrow{p_{3}}$ be the three-momenta of the three particles and let us introduce the new variables:

$$
\overrightarrow{\mathrm{Q}}=\overrightarrow{\mathrm{p}_{1}}+\overrightarrow{\mathrm{p}_{2}}+\overrightarrow{\mathrm{p}_{2}}, \quad \overrightarrow{\mathrm{k}_{1}}=\frac{\mathrm{m}_{3} \overrightarrow{\mathrm{p}}_{2}-\mathrm{m}_{2} \overrightarrow{\mathrm{p}}_{3}}{\mathrm{~m}_{2}+\mathrm{m}_{3}}, \overrightarrow{\mathrm{q}_{1}}=\frac{\left(\mathrm{m}_{2}+\mathrm{m}_{3}\right) \overrightarrow{\mathrm{p}}_{1}-\mathrm{m}_{1}\left(\overrightarrow{\mathrm{p}_{2}}+\overrightarrow{\mathrm{p}_{3}}\right)}{\mathrm{m}_{1}+\mathrm{m}_{2}+\mathrm{m}_{3}}
$$

and their cyclic permutations $\overrightarrow{\mathrm{k}}_{2}, \overrightarrow{\mathrm{q}}_{2}$ and $\overrightarrow{\mathrm{k}}_{3}, \overrightarrow{\mathrm{q}}_{3}$ 。 $Q$ is the total momentum, $\overrightarrow{\mathrm{k}}_{1}$ is the relative momentum between the particles 2 and 3 and $\overrightarrow{q_{1}}$ is the relative momentum of the particle 1 with respect to the cluster $2-3$. These variables are the most suitable ones for our purposes and any pair $\left\{\vec{k}_{i}, \vec{q}_{i}\right\}$ can be used for the description of the system. From now on we shall assume equal masses and $m=1$. For practical purposes, we write down some relations between the different variables

$$
\begin{array}{ll}
\vec{p}_{1}=\frac{1}{3} \overrightarrow{\mathrm{q}}+\overrightarrow{\mathrm{q}}_{1}, & \overrightarrow{\mathrm{p}_{2}}=\frac{1}{3} \overrightarrow{\mathrm{q}}-\frac{1}{2} \overrightarrow{\mathrm{q}}_{1}+\overrightarrow{\mathrm{k}}_{1}, \quad \overrightarrow{\mathrm{p}}_{3}=\frac{1}{3} \overrightarrow{\mathrm{Q}}-\frac{1}{2} \overrightarrow{\mathrm{q}}_{1}-\overrightarrow{\mathrm{k}}_{1} \\
\overrightarrow{\mathrm{q}}_{2}=-\frac{1}{2} \overrightarrow{\mathrm{q}_{1}}+\overrightarrow{\mathrm{k}}_{1}, \quad \overrightarrow{\mathrm{k}}_{2}=-\frac{3}{4} \overrightarrow{\mathrm{q}}_{1}-\frac{1}{2} \overrightarrow{\mathrm{k}}_{1} \\
\overrightarrow{\mathrm{q}}_{3}=-\frac{1}{2} \overrightarrow{\mathrm{q}}_{1}-\overrightarrow{\mathrm{k}}_{1}, \quad \overrightarrow{\mathrm{k}_{3}}=\frac{3}{4} \overrightarrow{\mathrm{q}_{1}}-\frac{1}{2} \overrightarrow{\mathrm{k}}_{1} \\
\overrightarrow{\mathrm{q}}_{1}+\overrightarrow{\mathrm{q}}_{2}+\overrightarrow{\mathrm{q}}_{3}=0
\end{array}
$$

We assume that the particle 1 only is charged; then the form factor reads:

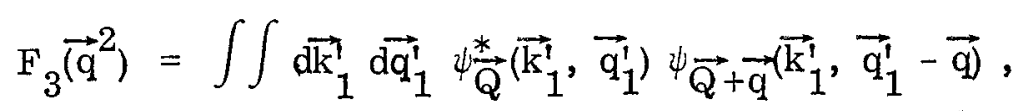

where $\psi=\psi^{1}+\psi^{2}+\psi^{3}$ and $\psi^{\mathbf{i}}$ are the Faddeev components satisfying the 
equation:

$$
\left(\begin{array}{c}
\vec{\psi}^{-1} \\
\psi^{2} \\
\psi^{3}
\end{array}\right)=-\mathrm{G}_{0}(\mathrm{E}) \quad\left(\begin{array}{ccc}
0 & \mathrm{~T}_{1}(\mathrm{E}) & \mathrm{T}_{1}(\mathrm{E}) \\
\mathrm{T}_{2}(\mathrm{E}) & 0 & \mathrm{~T}_{2}(\mathrm{E}) \\
\mathrm{T}_{3}(\mathrm{E}) & \mathrm{T}_{3}(\mathrm{E}) & 0
\end{array}\right) \quad\left(\begin{array}{c}
\psi^{1} \\
\psi^{2} \\
\psi^{3}
\end{array}\right)
$$

Here $G_{0}(E)=\frac{1}{\mathrm{H}_{0}-\mathrm{E}}$, where $\mathrm{H}_{0}$ is the free three-body Hamiltonian and $\mathrm{E}$ (the mass of the three-body ground state) is below any threshold. $\mathrm{T}_{i}(\mathrm{E})$ is the twobody scattering matrix between particles $\mathrm{j}$ and $\mathrm{k}(\mathrm{i} \neq \mathrm{j} \neq \mathrm{k})$ 。

In order to evaluate the asymptotic behaviors, we now introduce the "vertex function" $\varphi$ :

$$
\varphi=\varphi^{1}+\varphi^{2}+\varphi^{3}, \quad \psi^{\mathrm{i}}=\mathrm{G}_{0} \varphi^{\mathrm{i}}
$$

and we consider the once-iterated Faddeev equations:

$$
\begin{aligned}
& \varphi^{1}=\mathrm{T}_{1} \mathrm{G}_{0} \mathrm{~T}_{2} \mathrm{G}_{0} \varphi^{1}+\mathrm{T}_{1} \mathrm{G}_{0} \mathrm{~T}_{3} \mathrm{G}_{0} \varphi^{1}+\mathrm{T}_{1} \mathrm{G}_{0} \mathrm{~T}_{2} \mathrm{G}_{0} \varphi^{3}+\mathrm{T}_{1} \mathrm{G}_{0} \mathrm{~T}_{3} \mathrm{G}_{0} \varphi^{2} \\
& \varphi^{2}=\mathrm{T}_{2} \mathrm{G}_{0} \mathrm{~T}_{1} \mathrm{G}_{0} \varphi^{2}+\mathrm{T}_{2} \mathrm{G}_{0} \mathrm{~T}_{3} \mathrm{G}_{0} \varphi^{2}+\mathrm{T}_{2} \mathrm{G}_{0} \mathrm{~T}_{1} \mathrm{G}_{0} \varphi^{3}+\mathrm{T}_{2} \mathrm{G}_{0} \mathrm{~T}_{3} \mathrm{G}_{0} \varphi^{1} \\
& \varphi^{3}=\mathrm{T}_{3} \mathrm{G}_{0} \mathrm{~T}_{1} \mathrm{G}_{0} \varphi^{3}+\mathrm{T}_{3} \mathrm{G}_{0} \mathrm{~T}_{2} \mathrm{G}_{0} \varphi^{3}+\mathrm{T}_{3} \mathrm{G}_{0} \mathrm{~T}_{1} \mathrm{G}_{0} \varphi^{2}+\mathrm{T}_{3} \mathrm{G}_{0} \mathrm{~T}_{2} \mathrm{G}_{0} \varphi^{1}
\end{aligned}
$$

The first term of the first equation reads explicitly:

$$
\begin{aligned}
& \varphi \frac{1}{\mathrm{Q}^{2}}\left(\overrightarrow{\mathrm{k}}_{1}, \overrightarrow{\mathrm{q}}_{1}\right)=\iint \mathrm{d} \overrightarrow{\mathrm{k}}_{2}^{\prime} \mathrm{d \vec {q } _ { 2 } ^ { \prime }} \mathrm{t}_{1}\left(\overrightarrow{\mathrm{k}}_{1}, \overrightarrow{\mathrm{q}}_{2}^{\prime}+\frac{1}{2} \overrightarrow{\mathrm{q}}_{1} ; \mathrm{E}-\frac{3}{4} \overrightarrow{\mathrm{q}}_{1}^{2}-\frac{1}{6} \overrightarrow{\mathrm{Q}}^{2}\right) \frac{1}{\overrightarrow{\mathrm{q}}_{1}^{2}+\overrightarrow{\mathrm{q}}_{1} \cdot \overrightarrow{\mathrm{q}}_{2}^{\prime}+\overrightarrow{\mathrm{q}}_{2}^{\prime}-\mathrm{E}-\frac{1}{6} \overrightarrow{\mathrm{Q}}^{2}} \\
& \cdot t_{2}\left(-\vec{q}_{1}-\frac{1}{2} \vec{q}_{2}^{\prime}, \vec{k}_{2}^{\prime} ; E-\frac{3}{4} \vec{q}_{1}^{2}-\frac{1}{6} \vec{Q}^{2}\right) \frac{1}{\vec{q}_{2}^{\prime}+\vec{q}_{2}^{\prime} \cdot \vec{q}_{1}^{\prime}+\vec{q}_{1}^{2}-E-\frac{1}{6} \vec{Q}^{2}} \varphi \frac{1}{\vec{Q}^{2}}\left(\vec{q}_{1}^{\prime}, \vec{k}_{1}^{\prime}\right)+\ldots
\end{aligned}
$$


where $\left\{\overrightarrow{k_{1}^{t}}, \overrightarrow{q_{1}^{i}}\right\}$ and $\left\{\vec{k}_{2}^{t}, \vec{q}_{2}^{i}\right\}$ are related by Eq. (6). The high $\vec{q}^{2}$ behavior of the form factor (7) is given once we know the behavior of $\psi$ (or $\varphi$ ) for large $|\vec{q}|$ and $|\overrightarrow{\mathrm{k}}|$ (we always suppose that the low momenta do not create any trouble). In this region the $t$-matrix behaves as the potential up to logarithms so that, for the potential $(3), t\left(\vec{k}, \overrightarrow{k^{1}}\right) \simeq \frac{1}{\left|\vec{k}-\overrightarrow{k^{1}}\right|{ }^{1+\theta}} ;$ by means of a simple consistency argument we find that the only behavior consistent with Eq. (11) is given by

$$
\left.\varphi \stackrel{\frac{1}{\vec{Q}}}{\left(\vec{q}_{1}\right.}, \vec{k}_{1}\right)_{\left|\overrightarrow{k_{1}}\right| \rightarrow \infty} \underset{\left|\vec{q}_{1}\right| \rightarrow \infty}{\simeq} \frac{1}{\vec{q}_{1}^{2}}\left\{\frac{1}{\left|-\frac{1}{2} \vec{q}_{1}+\vec{k}_{1}\right|^{1+\theta}} \frac{1}{\left|\vec{q}_{1}\right|^{1+\theta}} \frac{1}{\left|\frac{1}{2} \vec{q}_{1}+\vec{k}_{1}\right|^{1+\theta}} \frac{1}{\left|\vec{q}_{1}\right|^{1+\theta}}\right\}
$$

We can obtain this result starting with a definite ansatz on the asymptotic behavior of the vertex function (for example the estimate $(7.36)$ of Ref.16). Because we are faced with an Euclidean metric, we can apply the Weinberg theorem ${ }^{21}$ and the asymptotic behaviors are simply given by a power counting. We find inconsistency, unless the ansatz is precisely the one given in formula (12). The behavior of $\varphi^{2}$ and $\varphi^{3}$ is easily found, so that from (9) and (12) we recover the following behavior for the wave function:

$$
\begin{aligned}
& \psi_{\vec{Q}}\left(\vec{q}_{1}, \vec{k}_{1}\right)=\frac{1}{\vec{k}_{1}^{2}+\frac{3}{4} \overrightarrow{\mathrm{q}}_{1}^{2}+\frac{1}{6} \overrightarrow{\mathrm{Q}}^{2}-\mathrm{E}} \varphi \overrightarrow{\vec{Q}}\left(\overrightarrow{\mathrm{q}_{1}}, \overrightarrow{\mathrm{k}_{1}}\right) \\
& \simeq \frac{1}{\vec{k}_{1}^{2}+\vec{q}_{1}^{2}}\left\{\frac{1}{\left|q_{1}\right|^{3+\theta}} \frac{1}{\left|-\frac{1}{2} \vec{q}_{1}+\vec{k}_{1}\right|^{1+\theta}}+\frac{1}{\left|\frac{1}{2} \vec{q}_{1}+\vec{k}_{1}\right|^{1+\theta}}\right]+\frac{1}{\left|-\frac{1}{2} \vec{q}_{1}+\vec{k}_{1}\right|^{3+\theta}} \\
& \left.\left[\frac{1}{\left|q_{1}\right|^{1+\theta}}+\frac{1}{\left|\frac{1}{2} \overrightarrow{q_{1}}+\vec{k}_{1}\right|^{1+\theta}}\right]+\frac{1}{\left|\frac{1}{2} \vec{q}_{1}+\vec{k}_{1}\right|^{3+\theta}}\left[\frac{1}{\left|q_{1}\right|^{1+\theta}}+\frac{1}{\left|-\frac{1}{2} \vec{q}_{1}+\vec{k}_{1}\right|^{1+\theta}}\right]\right\}
\end{aligned}
$$


The three terms which appear in Eq. (13) are easily understood. Equation (13), in fact, turns out to be symmetric in $\overrightarrow{q_{1}}, \vec{q}_{2}, \vec{q}_{3}$ and, consequently, in $\vec{p}_{1}, \vec{p}_{2}, \vec{p}_{3}$. By counting the powers in Eq. (13) and by observing that no dangerous region of integration exists (we could express everthing as a function of $\vec{p}_{1}, \vec{p}_{2}, \vec{p}_{3}$ ), it turns out that the wave function $\psi$ is integrable and

$$
\begin{aligned}
& \psi \overrightarrow{\mathrm{Q}}+\overrightarrow{\mathrm{q}}\left(\overrightarrow{\mathrm{k}}_{1}, \overrightarrow{\mathrm{q}}_{1}-\overrightarrow{\mathrm{q})} \underset{|\overrightarrow{\mathrm{q}}| \rightarrow \infty}{\simeq} \frac{1}{|\overrightarrow{\mathrm{q}}|^{6+2 \theta}}\right. \\
& \overrightarrow{\mathrm{k}}_{1}, \overrightarrow{\mathrm{q}}_{1} \text { fixed }
\end{aligned}
$$

From (7), (13) and (14), making use of the Weinberg theorem, ${ }^{21}$ we finally get:

$$
F_{3}\left(\vec{q}^{2}\right) \underset{|\vec{q}| \rightarrow \infty}{\simeq} \frac{1}{|\vec{q}|^{6+2 \theta}} \quad 0<\theta
$$

which has to be compared with $\mathrm{F}_{2}\left(\overrightarrow{\mathrm{q}}^{2}\right) \simeq \frac{1}{|\overrightarrow{\mathrm{q}}|^{3+\theta}}$ given in Eq. (4). The asymptotic behavior of the form factors, therefore, does depend on the number of the constituents (at least for 2 and 3 ). The slowest decrease we can achieve is $|\vec{q}|^{-3}$ and $|\vec{q}|^{-6}$ in the limiting case $\theta \rightarrow 0$.

It is interesting to remark that the three-body result is not affected by the existence or nonexistence of bound states in the two-body subchannels; in fact, for large momentum transfer, the two-body t-matrix is dominated by the scattering part and not by the discrete spectrum (cf. Ref. 16, Theorem 4.2).

In the second part of this section we shall discuss the case of separable potentials for reasons given above. Let us assume a separable contribution to the potential:

$$
\tilde{V}\left(\vec{k}, \overrightarrow{k^{t}}\right)=\lambda g(|\vec{k}|) g\left(\left|\overrightarrow{k^{t}}\right|\right)
$$

Furthermore, we shall assume that this part of the potential is dominating at short distances so that we can consider an interaction entirely described by the 
potential (16). The related $t$-matrix is given by the simple expression ${ }^{22}$

$$
t\left(\vec{k}, \overrightarrow{k^{\prime}} ; E\right)=g(|\vec{k}|) t(E) g(|\vec{k}|)
$$

where

$$
t(E)=\frac{1}{1+4 \pi \lambda \int_{0}^{\infty} d|\vec{q}| \frac{q^{2}|g(|\vec{q}|)|^{2}}{\vec{q}^{2}-E+i \epsilon}}
$$

In order that $t(E)$ may exist, we have to assume:

$$
g(|\vec{q}|) \underset{|\vec{q}|}{\simeq} \frac{1}{|\vec{q}|^{1 / 2+\theta}} \quad \theta>0
$$

Then, in the two-particle case, we immediately obtain from (1) and (2):

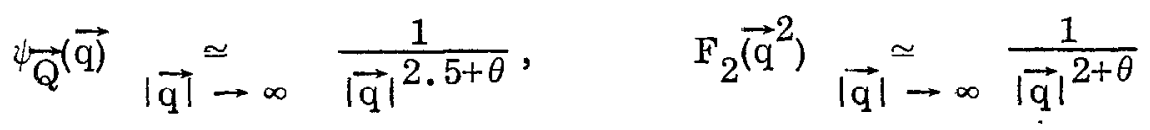

If we insert the t-matrix (17) in Eq. (8), we obtain the following simple structure of the three-body bound state wave function:

$$
\psi \vec{Q}\left(\vec{k}_{i}, \vec{q}_{i}\right)=\frac{g\left(\left|\overrightarrow{k_{i}}\right|\right) t\left(E-\frac{3}{4} \vec{q}_{i}^{2}-\frac{1}{6} \vec{Q}^{2}\right) \tilde{g}^{i}\left(\left|\overrightarrow{q_{i}}\right|\right)}{\vec{k}_{1}^{2}+\frac{3}{4} \vec{q}_{1}^{2}+\frac{1}{6} \vec{Q}^{2}-E}
$$

where the functions $\tilde{\mathrm{g}}^{\mathrm{i}}$ satisfy the (noniterated) coupled equations:

$$
\begin{aligned}
& \tilde{g}^{i}\left(\left|\overrightarrow{q_{i}}\right|\right)=\int d \vec{p} \frac{g\left(\left|\vec{p}+\frac{1}{2} \overrightarrow{q_{i}}\right|\right) t\left(E-\frac{3}{4} \vec{p}^{2}-\frac{1}{6} \vec{Q}^{2}\right) g\left(\left|\frac{1}{2} \vec{p}+\overrightarrow{q_{i}}\right|\right)}{\vec{q}_{i}^{2}+\overrightarrow{q_{i}} \cdot \vec{p}+\vec{p}^{2}-E-\frac{1}{6} \vec{Q}^{2}}\left[\tilde{g}^{j}(|\vec{p}|)+\tilde{g}^{k}(|\vec{p}|)\right] \\
& \mathrm{i} \neq \mathrm{j} \neq \mathrm{k}=1,2,3 \text {. }
\end{aligned}
$$

From the assumption (19) and the structure (18) of the t-matrix, it directly 
follows that the only behavior compatible with Eq. (22) is:

$$
g^{i}\left(\overrightarrow{q_{i}}\right) \simeq \frac{1}{\left|\overrightarrow{q_{i}}\right|^{3+2 \theta}}
$$

This leads to the asymptotic behavior of the wave function:

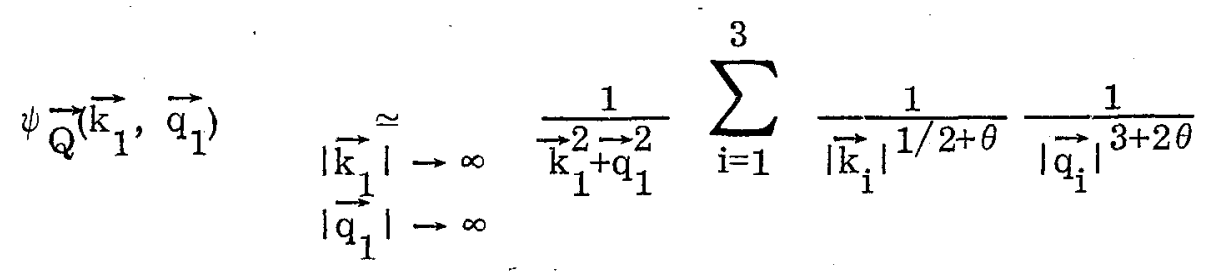

This wave function is not integrable but the same analysis we have applied in the local case still works and we obtain:

$$
\left.\mathrm{F}_{3} \overrightarrow{\mathrm{q}}^{2}\right) \simeq \frac{1}{|\overrightarrow{\mathrm{q}}|^{5+2 \theta}}
$$

Therefore, the $\mid \vec{q}^{-2}$ behavior of the two-body form factor is achieved with the potentials (16) in the limit $\theta \rightarrow 0$ without any dependence on the coupling constant. With the same limiting potential the three-body form factor behaves like $\mid \vec{q}^{-5}$.

\section{RELATIVISTIC MODELS}

\section{A. Two-Body}

Next we consider the asymptotic behavior of the form factor of relativistic two- and three-body bound states. Again, we only consider s-wave bound states and always assume that the masses are equal, $\mathrm{m}=1$, and only one particle is charged.

For the two-body bound state, the electromagnetic current in the ladder approximation is shown in Fig. 1 a and it can be written: 


$$
\begin{aligned}
\left\langle\psi\left|J_{\mu}\right| \psi\right\rangle= & 2 i \int d^{4} p \varphi_{Q}(p) \frac{1}{\left[\frac{1}{2} Q+p\right]^{2}-1}(Q+2 p+q)_{\mu} \frac{1}{\left[\frac{1}{2} Q-p\right]^{2}-1} \frac{1}{\left[\frac{1}{2} Q+p+q\right]^{2}-1} \\
& \cdot \varphi_{Q+q}(p+q / 2)
\end{aligned}
$$

where $\varphi_{Q}$ is the vertex function satisfying the Bethe-Salpeter equation (cf。Fig。1b):

$$
\varphi_{Q}(p)=(-i \lambda) \int d d^{4} k(p-k) G_{0}(k) \varphi_{Q}(k)
$$

and

$$
G_{0}(k)=\left[\left(\frac{1}{2} Q+k\right)^{2}-1\right]^{-1}\left[\left(\frac{1}{2} Q-k\right)^{2}-1\right]^{-1}
$$

We assume the interaction of the form:

$$
\mathrm{V}(\mathrm{p})=\int_{0}^{\infty} \mathrm{d} \mu^{2} \frac{\sigma\left(\mu^{2}\right)}{\left(\mathrm{p}^{2}+\mu^{2}\right)}, \quad \sigma\left(\mu^{2}\right) \underset{\mu^{2} \rightarrow \infty}{\simeq}\left(\mu^{2}\right)^{-\theta}, \quad 0<\theta \leq 1
$$

so that

$$
\mathrm{V}(\mathrm{p}) \underset{\mathrm{p}^{2} \rightarrow \infty}{\simeq}\left(\mathrm{p}^{2}\right)^{-\theta}
$$

Here, the $\lambda \varphi^{3}$ and the $\lambda \varphi^{4}$ theories are described by $\theta=1$ and $\theta=0$, respectively. By means of a simple consistency argument it is straightforward to derive from Eq. (27) the following asymptotic behavior of the two-body wave function: ${ }^{1-4}$

$$
\varphi_{Q}(p) \underset{p^{2} \rightarrow \infty}{\simeq}\left(p^{2}\right)^{-\theta}
$$

which, inserted in Eq. (26), gives for the form factor: ${ }^{1-4}$

$$
F_{2}\left(q^{2}\right) \underset{q^{2} \rightarrow \infty}{\simeq}\left(q^{2}\right)^{-1-\theta}
$$


From Eqs. (30) and (32) it follows that with a $\lambda \varphi^{3}$ theory we obtain $\mathrm{F}_{2}\left(\mathrm{q}^{2}\right) \simeq\left(\mathrm{q}^{2}\right)^{-2}$ whereas we reach the $1 / \mathrm{q}^{2}$ behavior in the limiting case $\theta \rightarrow 0$, $\dot{\theta}>\overrightarrow{0}$. For $\theta=0$, the consistency argument does not apply any longer and this reflects the well-known fact that in the $\lambda \varphi^{4}$ theory, which corresponds to the case $\theta=0$, the large momentum transfer behavior of the form factor depends on the coupling constant ${ }^{2,4,18}$ (cf. the potential $\left(-\frac{\lambda}{r^{2}}\right)$ in Section II).

The use of the parameter $\theta$ in the definition of the potential is essentially the procedure applied in the analytic regularization; ${ }^{23}$ on account of the possible nonanalitic dependence of the renormalizable theories on this parameter, however, we should not be surprised at this discontinuity.

\section{B. Three-Body}

For the three-body case we shall assume a pair-wise interaction between the constituents and we shall consider the ladder graphs given in Fig. 2 only.

We make the Faddeev decomposition of the bound state vertex function, i.e., $\varphi=\varphi^{1}+\varphi^{2}+\varphi^{3}$ where $\varphi^{1}$ is related to all the graphs in which the interaction between particles 2 and 3 comes first. Graphically, the once iterated relativistic Faddeev equations are shown in Fig. 3, where the zig-zag lines stay for a two-body t-matrix with a three-body propagator (cf. Fig. 4). It is easy to see that the iteration of Fig. 3 reproduces all the (uncrossed) ladder graphs. The integral equation for the vertex function can be written in a symbolical form similar to the nonrelativistic equation (Eq。(10)):

$$
\begin{aligned}
& \varphi^{1}=\mathrm{T}_{1} \mathrm{G}_{0} \mathrm{~T}_{2} \mathrm{G}_{0} \varphi^{1}+\mathrm{T}_{1} \mathrm{G}_{0} \mathrm{~T}_{3} \mathrm{G}_{0} \varphi^{1}+\mathrm{T}_{1} \mathrm{G}_{0} \mathrm{~T}_{2} \mathrm{G}_{0} \varphi^{3}+\mathrm{T}_{1} \mathrm{G}_{0} \mathrm{~T}_{3} \mathrm{G}_{0} \varphi^{2} \\
& \varphi^{2}=\mathrm{T}_{2} \mathrm{G}_{0} \mathrm{~T}_{1} \mathrm{G}_{0} \varphi^{2}+\mathrm{T}_{2} \mathrm{G}_{0} \mathrm{~T}_{3} \mathrm{G}_{0} \varphi^{2}+\mathrm{T}_{2} \mathrm{G}_{0} \mathrm{~T}_{1} \mathrm{G}_{0} \varphi^{3}+\mathrm{T}_{2} \mathrm{G}_{0} \mathrm{~T}_{3} \mathrm{G}_{0} \varphi^{1} \\
& \varphi^{3}=\mathrm{T}_{3} \mathrm{G}_{0} \mathrm{~T}_{1} \mathrm{G}_{0} \varphi^{3}+\mathrm{T}_{3} \mathrm{G}_{0} \mathrm{~T}_{2} \mathrm{G}_{0} \varphi^{3}+\mathrm{T}_{3} \mathrm{G}_{0} \mathrm{~T}_{1} \mathrm{G}_{0} \varphi^{2}+\mathrm{T}_{3} \mathrm{G}_{0} \mathrm{~T}_{2} \mathrm{G}_{0} \varphi^{1}
\end{aligned}
$$


where now, symbolically:

$$
\left.<p_{1} p_{2} p_{3}\left|T_{1}\right| p_{1}^{\prime} p_{2}^{\prime} p_{3}^{\prime}\right\rangle=\left(p_{1}^{2}+1\right) \delta^{4}\left(p_{1}-p_{1}^{\prime}\right) \delta^{4}\left(p_{2}+p_{3}-p_{2}^{\prime}-p_{3}^{\prime}\right) t_{1}\left(p_{2}, p_{3} ; p_{2}^{\prime}, p_{3}^{\prime}\right) 。
$$

Here, $t_{1}$ is the usual two-body Bethe-Salpeter scattering matrix between particles 2 and 3 in the ladder approximation, and:

$$
\left\langle\mathrm{p}_{1} \mathrm{p}_{2} \mathrm{p}_{3}\left|\mathrm{G}_{0}\right| \mathrm{p}_{1}^{\prime} \mathrm{p}_{2}^{\prime} \mathrm{p}_{3}^{\prime}\right\rangle=\frac{1}{\mathrm{p}_{1}^{2}-1} \frac{1}{\mathrm{p}_{2}^{3}-1} \frac{1}{\mathrm{p}_{3}^{2}-1} \delta^{4}\left(\mathrm{p}_{1}-\mathrm{p}_{1}^{\prime}\right) \delta^{4}\left(\mathrm{p}_{2}-\mathrm{p}_{2}^{\prime}\right) \delta^{4}\left(\mathrm{p}_{3}-\mathrm{p}_{3}^{\prime}\right)
$$

(Relativistic Faddeev equations have been written down by many authors in different approximations. See for example Refs. (12) $-(15)$ and (20).)

As we did in the nonrelativistic case, we introduce the four-momenta:

$$
\mathrm{Q}=\mathrm{p}_{1}+\mathrm{p}_{2}+\mathrm{p}_{3}, \quad \mathrm{k}_{1}=\frac{1}{2}\left(\mathrm{p}_{2}-\mathrm{p}_{3}\right), \quad \mathrm{q}_{1}=\frac{2 \mathrm{p}_{1}-\left(\mathrm{p}_{2}+\mathrm{p}_{3}\right)}{3}
$$

and their cyclic permutations $r_{2}, q_{2}$ and $r_{3}, q_{3}$ (the relation among them is the same as given in Eq. (16)). The Eq. (33) now reads explicitly:

$$
\begin{aligned}
& \varphi_{Q}^{1}\left(k_{1}, q_{1}\right)=\iint d k^{\prime} d q^{\prime} t_{1}\left(\frac{1}{3} Q-\frac{1}{2} q_{1}+k_{1}, \frac{1}{3} Q-\frac{1}{2} q_{1}-k_{1} ; \frac{1}{3} Q-\frac{1}{2} q^{\prime}+k^{\prime}, \frac{1}{3} Q+\frac{1}{2} q^{\prime}-k^{\prime}-q_{1}\right) \\
& {\left[\left(\frac{1}{3} Q-\frac{1}{2} q^{\prime}+k^{\prime}\right)^{2}-1\right]^{-1}\left[\left(\frac{1}{3} Q+\frac{1}{2} q^{\prime}-k^{\prime}-q_{1}\right)^{2}-1\right]^{-1}} \\
& t_{2}\left(\frac{1}{3} Q+q_{1}, \frac{1}{3} Q+\frac{1}{2} q^{\prime}-k^{\prime}-q_{1} ; \frac{1}{3} Q+q^{\prime}, \frac{1}{3} Q-\frac{1}{2} q^{\prime}-k^{\prime}\right) \\
& \cdot\left[\left(\frac{1}{3} Q+q^{\prime}\right)^{2}-1\right]^{-1}\left[\left(\frac{1}{3} Q-\frac{1}{2} q^{\prime}-k^{\prime}\right)^{2}-1\right]^{-1} \varphi_{Q}^{1}\left(k^{\prime}, q^{\prime}\right)+\iint \ldots \varphi^{1}+\iint \ldots \varphi^{2}+\iint \ldots \varphi^{3} . \\
& \varphi_{Q}^{2}\left(k_{2}, q_{2}\right)=\ldots \\
& \varphi_{Q}^{3}\left(k_{3}, q_{3}\right)=\ldots
\end{aligned}
$$


In terms of the vertex function $\varphi=\varphi^{1}+\varphi^{2}+\varphi^{3}$, the electromagnetic current for the three-body bound state now reads (cf. Fig. 5):

$$
\begin{aligned}
& \left\langle\psi\left|J_{\mu}\right| \psi\right\rangle=\iint \mathrm{dq}_{1}^{\prime} \mathrm{dk}_{1}^{\prime} \varphi_{\mathrm{Q}}\left(\mathrm{k}_{1}^{\prime}, \mathrm{q}_{1}^{\prime}\right) \quad\left[\left(\frac{1}{2} \mathrm{Q}+\mathrm{q}_{1}^{\prime}\right)^{2}-1\right]^{-1}\left(\frac{2}{3} \mathrm{Q}+2 \mathrm{q}_{1}^{\prime}+\mathrm{q}\right)_{\mu} \\
& {\left[\left(\frac{1}{3} \mathrm{Q}-\frac{1}{2} \mathrm{q}_{1}^{\prime}+\mathrm{k}_{1}^{\prime}\right)^{2}-1\right]^{-1}\left[\left(\frac{1}{3} \mathrm{Q}-\frac{1}{2} \mathrm{q}_{1}^{\prime}-\mathrm{k}_{1}^{\prime}\right)^{2}-1\right]^{-1}\left[\left(\frac{1}{3} \mathrm{Q}+\mathrm{q}_{1}^{\prime}+\mathrm{q}\right)^{2}-1\right]^{-1}} \\
& \varphi_{\mathrm{Q}+\mathrm{q}}\left(\mathrm{k}_{1}^{\prime}, \mathrm{q}_{1}^{\prime}+\frac{2}{3} \mathrm{q}\right) .
\end{aligned}
$$

In order to evaluate the asymptotic behavior of the form factor, we need the behavior of the vertex function for large momenta. In this limit, the t-matrix reduces to the potential up to logarithms and the asymptotic integral equation reads:

$$
\begin{aligned}
& \varphi_{Q}^{1}\left(k_{1}, q_{1}\right) \simeq \iint d q^{\prime} d k^{\prime}\left[\left(\frac{1}{2} q_{1}-k_{1}-\frac{1}{2} q^{\prime}+k^{\prime}\right)^{2}\right]^{-\theta}\left[\left(\frac{1}{3} Q-\frac{1}{2} q^{\prime}+k^{\prime}\right)^{2}\right]^{-1} \\
& {\left[\left(\frac{1}{3} Q+\frac{1}{2} q^{\prime}-k^{\prime}-q_{1}\right)^{2}\right]^{-1}\left[\left(q_{1}-q^{\prime}\right)^{2}\right]^{-\theta}\left[\left(\frac{1}{3} Q+q^{\prime}\right)^{2}\right]^{-1}\left[\left(\frac{1}{3} Q-\frac{1}{2} q^{\prime}-k^{\prime}\right)^{2}\right]^{-1}} \\
& \varphi_{Q}^{1}\left(k^{\prime}, q^{\prime}\right)+\iint \ldots \varphi^{1}+\iint \ldots \varphi^{2}+\iint \ldots \varphi^{3} \\
& \varphi_{Q}^{2}\left(k_{2}, q_{2}\right) \simeq \ldots \\
& \varphi_{Q}^{3}\left(k_{3}, q_{3}\right) \simeq \ldots
\end{aligned}
$$

If we first consider the integration over a finite volume, we immediately obtain the following behavior:

$$
\begin{aligned}
\varphi_{Q}^{1}\left(q_{1}, k_{1}\right) & \simeq\left[\left(\frac{1}{2} q_{1}-k_{1}\right)^{2}\right]^{-\theta}\left[q_{1}^{2}\right]^{-1}\left[q_{1}^{2}\right]^{-\theta}+\ldots \\
& =2\left[\left(\frac{1}{2} q_{1}-k_{1}\right)^{2}\right]^{\theta}\left[q_{1}^{2}\right]^{-1}\left[q_{1}^{2}\right]^{-\theta}+2\left[\left(\frac{1}{2} q_{1}+k_{1}\right)^{2}\right]^{-\theta}\left(q_{1}^{2}\right)^{-1}\left[q_{1}^{2}\right]^{-\theta}
\end{aligned}
$$


When the integration variables are big, we substitute this ansatz in Eq。(39) and evaluate the contribution to the asymptotic behavior coming from the other regions of integration: $\mathrm{k}_{\mu}^{\prime}$ small and $\mathrm{q}_{\nu}^{\prime}=\mathrm{O}\left(\mathrm{q}_{1}\right)$ or $\mathrm{q}_{\mu}^{\prime}=\mathrm{O}\left(\frac{1}{2} \mathrm{q}_{1}-\mathrm{k}_{1}\right)$ and viceversa; $\mathrm{q}_{\mu}^{\prime}=\mathrm{O}\left(\mathrm{q}_{1}\right)$ and $\left(\frac{1}{2} \mathrm{q}^{\prime}-\mathrm{k}^{\prime}\right)_{\nu}=\mathrm{O}\left(\frac{1}{2} \mathrm{q}_{1}-\mathrm{k}_{1}\right)$ etc. The behavior (40) turns out to be dominant. Collecting from Eq. (39) the missing terms, we obtain for the vertex function (cf. (13)):

$$
\begin{aligned}
\varphi_{\mathrm{Q}}\left(\mathrm{q}_{1}, \mathrm{k}_{1}\right) & \simeq\left[\mathrm{q}_{1}^{2}\right]^{-1-\theta}\left\{\left[\left(\frac{1}{2} \mathrm{q}_{1}-\mathrm{k}_{1}\right)^{2}\right]^{-\theta}+\left[\left(\frac{1}{2} \mathrm{q}_{1}+\mathrm{k}_{1}\right)^{2}\right]^{-\theta}\right\} \\
& +\left[\left(\frac{1}{2} \mathrm{q}_{1}-\mathrm{k}_{1}\right)^{2}\right]^{-1-\theta}\left\{\left[\mathrm{q}_{1}^{2^{-}-\theta}+\left[\left(\frac{1}{2} \mathrm{q}_{1}+\mathrm{k}_{1}\right)^{2}\right]^{-\theta}\right\}\right. \\
& +\left[\left(\frac{1}{2} \mathrm{q}_{1}+\mathrm{k}_{1}\right)^{2}\right]^{-1-\theta}\left\{\left[\mathrm{q}_{1}\right]^{-\theta}+\left[\left(\frac{1}{2} \mathrm{q}_{1}-\mathrm{k}_{1}\right)^{2}\right]^{-\theta}\right\}
\end{aligned}
$$

From Eq. (41) it follows that the wave function:

$$
\begin{aligned}
\psi= & \mathrm{G}_{0} \varphi \rightarrow \psi_{\mathrm{Q}}\left(\mathrm{q}_{1}, \mathrm{k}_{1}\right)=\left[\left(\frac{1}{2} \mathrm{Q}+\mathrm{q}_{1}\right)^{2}-1\right]-1\left[\left(\frac{1}{3} \mathrm{Q}-\frac{1}{2} \mathrm{q}_{1}+\mathrm{k}_{1}\right)^{2}-1\right]^{-1} \\
& {\left[\left(\frac{1}{3} \mathrm{Q}-\frac{1}{2} \mathrm{q}_{1}-\mathrm{k}_{1}\right)^{2}-1\right]^{-1} \varphi_{\mathrm{Q}}\left(\mathrm{q}_{1}, \mathrm{k}_{1}\right) }
\end{aligned}
$$

is integrable. Furthermore:

$$
\varphi_{Q+q}\left(k_{1}, q_{1}+\frac{2}{3} q\right) \underset{\substack{q^{2} \rightarrow \infty \\ k_{1}, q_{1} \text { fixed }}}{\simeq}\left(q^{2}\right)^{-1-2 \theta}
$$

In order to evaluate the asymptotic behavior of the form factor (38), we first consider a finite region of integration and from Eq. (43) we obtain:

$$
F\left(q^{2}\right) \underset{q^{2} \rightarrow \infty}{\simeq}\left(q^{2}\right)^{-2-2 \theta}
$$


The other regions of integration confirm the behavior (44) as the dominant one. The nucleon form factor would correspond to the limiting case $\theta \rightarrow 0$ as it was necessary to consider in the two-body case in order to recover the correct pion form factor. So far, we have a consistency (although spinless) model for the pion and the nucleon form factors.

\section{ACKNOWLEDGMENTS}

It is a pleasure to thank R. Blankenbecler, S. J. Brodsky, M. Chanowitz and P. Zerwas for useful discussions and R. Blankenbecler for reading the manuscript. We also thank S. D. Drell for his warm hospitality at SLAC. 


\section{REFERENCES}

1. J.S. Ball and F. Zachariasen, Phys. Rev. 170, 1541 (1968).

2. M. Ciafaloni and P. Menotti, Phys. Rev. 173, 1575 (1968).

3. D. Amati, L. Caneschi and R. Jengo, Nuovo Cimento, 58, 783 (1968).

4. M. Ciafaloni, Phys. Rev. 176, 1898 (1968)。

5. M. Böhm, H. Joos and M. Krammer, Nuovo Cimento $\underline{7}$ A, 21 (1972).

6. R.F. Meyer, "Relativistic Three-Particle Problem and Heavy Quarks," DESY $73 / 42$, DESY preprint.

7. See for example, V. Silvestrini in: "Proceedings of the XVI International Conference on High Energy Physics," Vol。4, NAL (1972).

8. S. Brodsky and G. Farrar, Phys. Rev. Letters 31, 1153 (1973).

9. V.A. Matveev, R.M. Muradyan and A.N. Tavkhelidze, Nuovo Cimento Lettere, 7, 719 (1973).

10. C. Alabiso and G. Schierholz, to be published.

11. H. Lipkin, "Quarks for Pedestrians," Weizmann Institute preprint, WIS $-73 / 8-\mathrm{Ph}$.

12. A.A. Sogunov and A.N. Tavkhelidze, Nuovo Cimento 29, 380 (1963).

13. R. Blankenbecler and R. Sugar, "Proceedings of the 12th Annual International Conference on High Energy Physics at Dubna, 1964," Atomizdat, Moskow (1965) and Phys. Rev. 142, 1051 (1966).

14. V.A. Alessandrini and R.L. Omnes, Phys. Rev. 139 B, 167 (1965).

15. V.M. Vinogradov, Sov. J. of Nucl. Phys. 14, 609 (1972).

16. L.D. Faddeev, "Mathematical Aspects of the Three-Particle Problem in Quantum Scattering Theory," Jerusalem 1965.

17. L.D. Landau and E.M. Lifschitz, "Quantum Mechanics," Addison Wesley (1958) p. 35. 
18. A. Bastai, L. Bertocchi, S. Fubini, G. Furlan and M. Tonin, Nuovo Cimento $\underline{30}, 1512(1963)$.

19. S. D. Drell, A.C. Finn and M.H. Goldhaber, Phys. Rev. 157, 1402 (1967).

20. D. Stoyanov and A.N. Tavkhelidze, Phys. Letters $\underline{13}, 76$ (1964); V.P. Shelest and D. Stoyanov, Phys. Letters $\underline{13}, 256$ (1964).

21. S. Weinberg, Phys. Rev. 118, 838 (1960); cf. J.D. Bjorken and S. D. Drell, "Relativistic Quantum Field," McGraw-Hill, 1965, paragraph 19-10.

22. See for example, C. Lovelace, Phys. Rev. $\underline{135}$ B, 1225 (1964).

23. E. Speer, J. of Math. Phys. $\underline{9}, 1403$ (1968).

24. S. D. Drell and T.D. Lee, Phys. Rev. D $\underline{5}, 1738$ (1972). 


\section{FIGURE CAPTIONS}

1. The electromagnetic form factor in the ladder approximation for a two body bound state (1a). The Bethe-Salpeter equation in the ladder approximation for the wave (vertex) function of a two-body bound state (1b). The elementary two-body interaction is defined in formula (29).

2. The general graph in the ladder approximation for a system of three particles interacting with a two-body interaction.

3. The once-iterated relativistic Faddeev equations for the wave (vertex) function of a three-body bound state. The symbol $\Longrightarrow$ i means the Faddeev components $\psi^{i}\left(\varphi^{i}\right)$. The twisted lines represent the two-body Bethe-Salpeter T-matrix in the ladder approximation.

4. The two-body Bethe-Salpeter T-matrix with a three-particle propagator.

5. The electromagnetic form factor in the ladder approximation for a three-body bound state. 


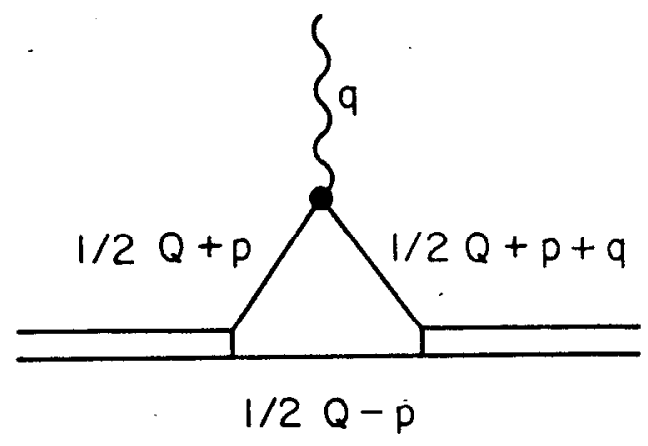

(a)

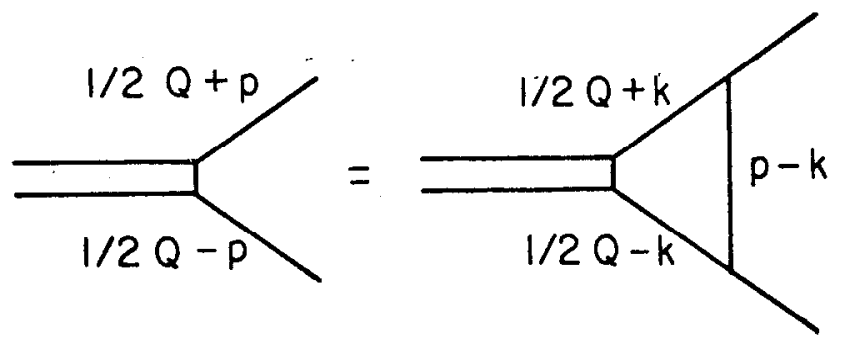

(b)

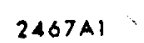

Fig. 1
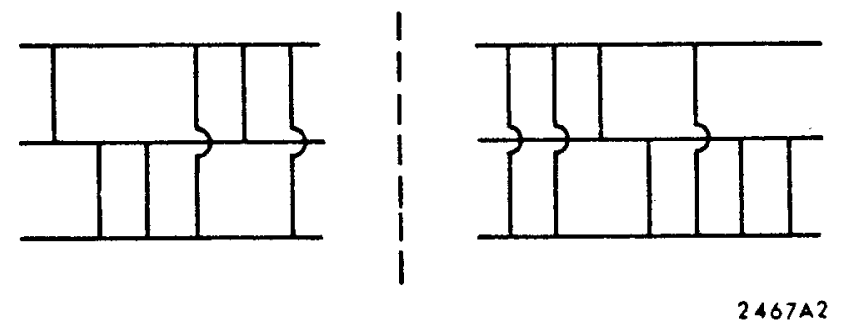

Fig. 2 

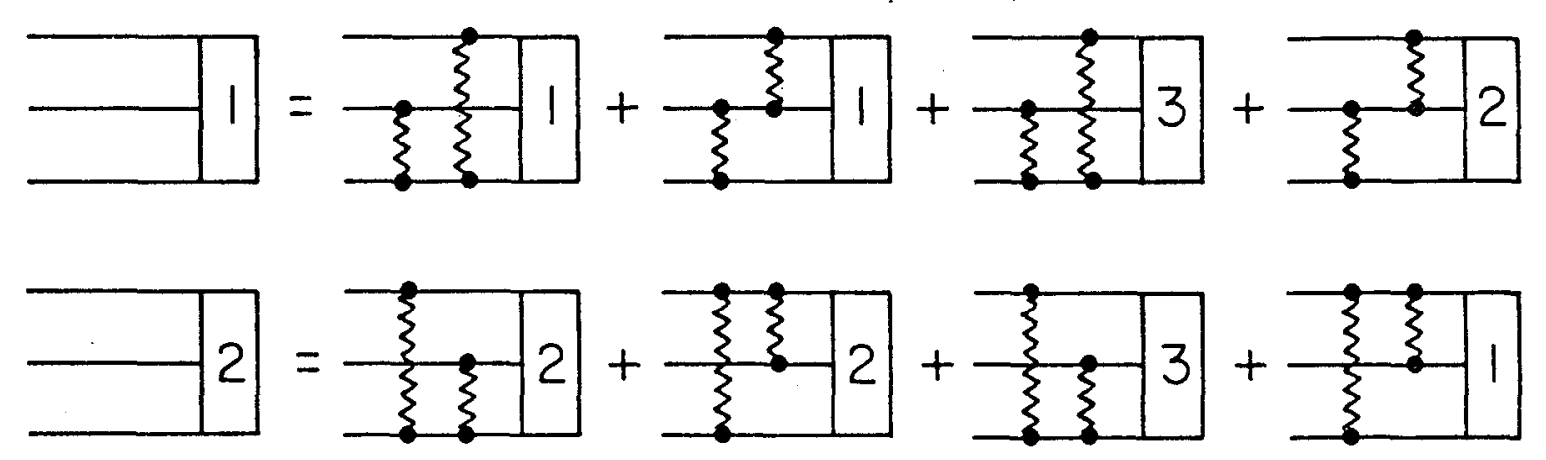

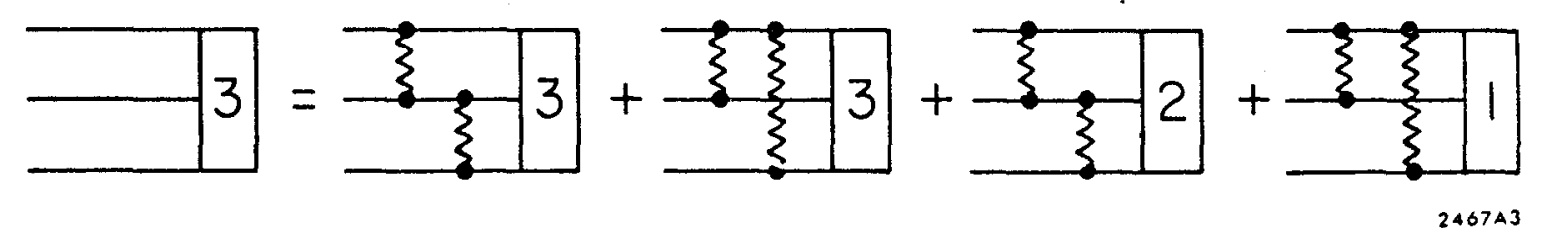

Fig. 3 


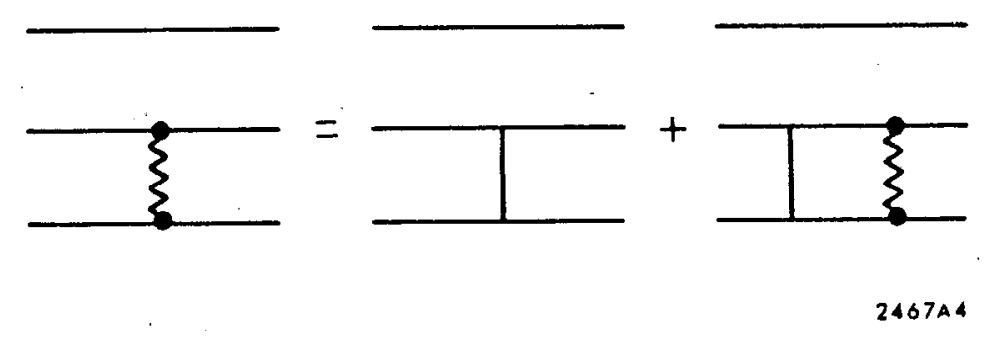

Fig. 4

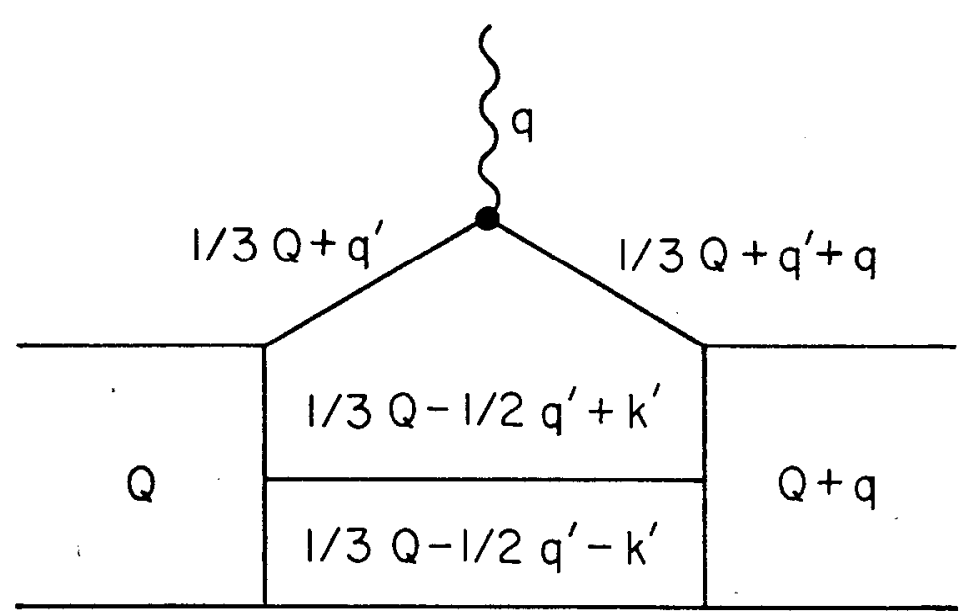

2467A5

Fig. 5 